\title{
DETERMINAÇÃO DO EQUILÍBRIO LÍQUIDO-LÍQUIDO DO SISTEMA BIODIESEL DE SOJA + METANOL + GLICEROL + ÁGUA A 333,15 K.
}

\author{
M. A. TEOTÔNIO, A. C. C. de MELO, L. STRAGEVITCH, L. DANIELSKI. \\ Universidade Federal de Pernambuco, Departamento de Engenharia Química \\ E-mail para contato: marcelio.qi2011@ hotmail.com
}

\begin{abstract}
RESUMO - Atualmente, há uma grande intensificação nos estudos e desenvolvimento de tecnologias mais limpas, visando minimizar as emissões de gás carbônico na atmosfera e redução significativa do uso do petróleo como principal fonte energética. Dentre estas tecnologias, encontra-se a utilização de biocombustíveis como fonte de energia renovável; um desses exemplos é o biodiesel. Como resultado da reação de transesterificação há a formação de biodiesel e glicerol. Estas fases se separam até que se atinja o estado de equilíbrio entre elas. A determinação dos dados de equilíbrio são imprescindíveis para o desenvolvimento e aperfeiçoamento dos equipamentos. O objetivo deste trabalho relaciona-se à medição experimental dos dados de equilíbrio líquido-líquido (ELL) do sistema biodiesel de soja (1) + metanol (2) + glicerol (3) + água (4) a 333,15 K e a pressão atmosférica. Adicionalmente, a predição e a correlação dos dados de equilíbrio foi realizada através do modelo termodinâmico UNIQUAC.
\end{abstract}

\section{INTRODUÇÃO}

Com iniciativa abordada no protocolo de Kyoto em 1997, criou-se um tratado internacional com compromissos mais rígidos para a redução da emissão dos gases que provocam o efeito estufa e com isso a preocupação com o meio ambiente vem aumentando desde então.

Os biocombustíveis têm recebido atenção em diversos países nos últimos anos devido às necessidades cada vez maiores de fontes de energia e diminuição nas emissões de gases poluentes. A utilização de biocombustíveis tornou-se uma alternativa para substituição de outros combustíveis. Dentre esses biocombustíveis surge como alternativa o biodiesel por apresentar propriedades semelhantes ao óleo diesel, dispensando modificações nos motores do ciclo diesel. Desde a crise do petróleo em 1970, o rápido aumento dos preços e incertezas quanto a disponibilidade petróleo, a preocupação crescente do meio ambiente e do efeito de gases de efeito estufa durante as últimas décadas, reavivou mais e mais interesse na utilização de óleos vegetais, como um substituto de combustível fóssil (WANG et al, 2006). 


\section{9 a 22 de outubro de 2014 \\ Florianópolis/SC}

O uso do biodiesel apresenta vantagens significativas. Na combustão, o biocombustível emite quantidades reduzidas de monóxido de carbono, materiais particulados, e dióxido de carbono $\left(\mathrm{CO}_{2}\right)$. O biodiesel não emite o dióxido de enxofre durante a queima por não conter enxofre em sua fórmula. Em comparação ao diesel, possui um ponto de fulgor mais alto, tornando-o assim mais seguro para o transporte e armazenamento (BALAT, 2008).

Conhecer as condições de equilíbrio líquido-líquido envolvidas na produção deste biodiesel é essencial para o melhor entendimento do processo de purificação (Negi et al., 2006). Levando em consideração a influência da água na etapa de purificação do biodiesel, esse estudo avaliou o comportamento de um sistema pseudo-quaternário biodiesel (1) + metanol (2) + glicerol (3) + água (4) em diferentes níveis de temperatura a pressão ambiente. A água, além de promover a hidrólise do biodiesel resultando em ácidos graxos livres, também está associada à proliferação de micro-organismos e à corrosão em tanques de estocagem com deposição de sedimentos. (LÔBO; FERREIRA; CRUZ; 2009).

\section{MATERIAIS E METÓDOS}

O biodiesel utilizado neste trabalho foi produzido a partir de óleo de soja refinado. $\mathrm{O}$ mesmo foi produzido no Laboratório de Combustível da UFPE. O sistema para medição dos dados experimentais do ELL para a mistura biodiesel (1) + glicerol (2) + metanol (3) + água (4) foi constituído por células de equilíbrio com encamisamento para circulação de fluido feita em vidro com volume de $100 \mathrm{~mL}$ e tampa de teflon para colocação do agitador mecânico e do sensor de temperatura. As células de ELL possuem dois amostradores pelos quais as alíquotas são retiradas de cada uma das fases. Agitadores mecânicos com velocidade de agitação de (200 a 2000) rpm, marc IKA®, modelo RW 20 digital foram utilizados. Termopares do tipo PT-100 com calibração RBC na faixa de $(293,15$ a 353,15$) \mathrm{K}$ acoplado a banho termostático foram responsáveis pelo controle da temperatura, o sistema descrito é o mesmo utilado por MAIA 2010. Biodiesel (1) + metanol (2) + glicerol (3) + água (4) foram preparadas gravimetricamente. Os componentes da mistura foram pesados diretamente na célula de ELL. As pesagens foram realizadas em uma balança semi-analítica. Após a pesagem dos componentes, a célula de ELL foi vedada e conectada a um banho termostático. Foi considerada a temperatura de 333,15 K. O fluido utilizado no banho foi a água. A temperatura na mistura presente no interior das células de equilíbrio foi medida com um termopar do tipo PT100.

Agitadores mecânicos foram usados para promover a mistura entre as fases por um período de uma hora e posteriormente foi mantido em repouso para a separação das duas fases. Após o equilíbrio atingido, amostras de cada fase foram coletadas com seringa de vidro.

As quantidades de cada componente necessárias para que ao final da separação, a interface ficasse aproximadamente no centro da célula, podem ser determinadas através de um sistema de equações conforme apresentado por Maduro (2005) e adaptadas para o sistema pseudoquaternário. 


$$
V_{i}=\frac{V}{2} M_{i}\left[\frac{x_{i}^{F O}}{\sum_{i=1,2,3,4}^{4} \frac{x_{i}^{F O_{M}}}{\rho_{i}}}+\frac{x_{i}^{F A}}{\sum_{i=1,2,3,4}^{4} \frac{x_{i}^{F A_{M}}}{\rho_{i}}}\right] \quad i=1,2,3,4
$$

nas quais $V$ é o volume da célula, $x$ é a fração molar, $M$ é a massa molar, $F O$ é fase rica em biodiesel e $F A$ é a fase rica em glicerol, $i$ faz referência a cada componente do sistema.

A determinação da composição de cada uma das fases foi realizada através de cromatografia gasosa com metodologia implementada em cromatógrafo com detector de ionização de chama utilizando uma coluna capilar cromatográfica VB-1 (ValcoBond) fase estacionária $100 \%$ dimetilpolisiloxano com comprimento de $30 \mathrm{~m}$, diâmetro interno de 0,32 mm e espessura do filme de $0,3 \mu \mathrm{m}$. Foi empregado um injetor tipo split/splitless e um detector de ionização de chama (Flame Ionization Detector - FID). As temperaturas do injetor e do detector foram de 523,15 e 543,15 K, respectivamente e à coluna foi aplicada a seguinte rampa de temperatura: $343,15 \mathrm{~K}$ durante 6 minutos, em seguida a temperatura foi elevada até $543,15 \mathrm{~K}$ à uma taxa de $20 \mathrm{~K} / \mathrm{min}$, permanecendo em $543,15 \mathrm{~K}$ pelo período de 24 minutos e 30 segundos. Hidrogênio foi usado como gás de arraste.

Para determinar a fração mássica de cada amostra de ELL, foram construídas curvas analíticas de calibração contendo os quatro componentes de interesse em diferentes concentrações. Para quantificação dos componentes presentes na amostra foi utilizado o fator de resposta, juntamente com o metil heptadecanoato $\left(C_{17}\right)$ como padrão interno. Foi construída uma curva para a fase rica em éster e outra para a fase rica em glicerol. Isopropanol foi utilizado como solvente para diluição e homogeneização das amostras.

Os dados de ELL experimentais foram correlacionados utilizando os modelos UNIQUAC. Para o modelo UNIQUAC foi empregada a metodologia proposta por Stragevitch e d'Ávila (1997) para a obtenção dos parâmetros do modelo a partir de correlação dos dados empíricos a $333,15 \mathrm{~K}$.

\section{RESULTADOS E DISCUSSÕES}

Os dados de equilíbrio ELL estão apresentados com base livre de água, levando em consideração a pequena quantidade de água que foi adicionada ao sistema. Assim, foi analisada a influência de pequenas quantidades de água presentes na produção do biodiesel.

A correlação de OTHMER E TOBIAS (1942) foi utilizada para testar a validade dos dados experimentais do sistema estudado. Autores como MAIA et al. (2011), ARDILA et al. (2012), FRANÇA et al. (2013), utilizaram o método de correlação para medir a confiabilidade dos seus dados obtidos experimentalmente. A correlação consiste em plotar o gráfico do $\log ((1-a) / a)$ contra o $\log ((1-b) / b)$, onde $a$ é a fração molar de biodiesel na fase rica em biodiesel e $b$ é a fração molar de glicerol na fase rica em glicerol. 
A partir das frações mássicas $(w)$ determinadas através da cromatografia gasosa, a fração molar pode ser determinada através do uso da equação (2). Para construção dos gráficos de Othmer-Tobias, a fração molar dos componentes foi calculada, na qual $w_{i}$ é a fração mássica e $M_{i}$ é a massa molar do componente $i$.

$$
x_{i}=\frac{\frac{w_{i}}{M_{i}}}{\sum \frac{w_{i}}{M_{i}}}
$$

No diagrama de Othmer-Tobias apresentado na Figura 1 pode-se observar a consistência dos resultados das linhas de amarração para o sistema pseudo-quaternários biodiesel (1) + metanol (2) + glicerol (3) na temperatura 333,15 K, o valor do parâmetro de ajuste linear encontrado foi de 0,9872 .

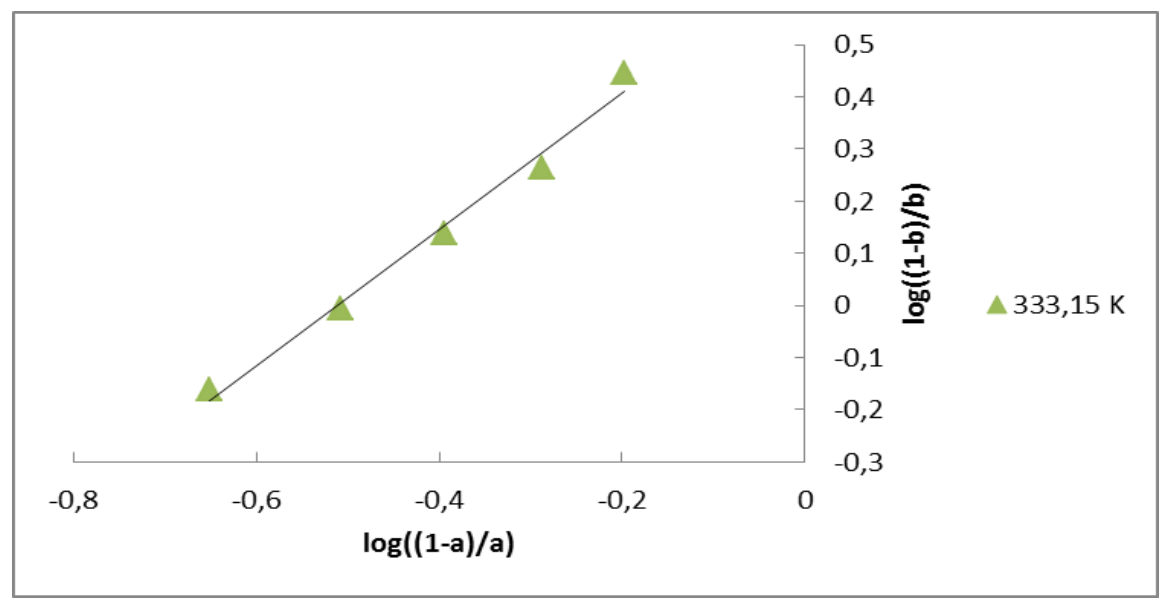

Figura 1 Gráfico Othmer-Tobias para o sistema biodiesel (1) + metanol (2) + glicerol (3) + água (4) a $333,15 \mathrm{~K}$

A distribuição do álcool entre as fases ricas em biodiesel e glicerol foram calculadas nos sistemas em estudo. Assim, os valores dos coeficientes de distribuição $\mathrm{K}_{2}$ do metanol foram calculados pela relação:

$$
K_{2}=\frac{w_{2}^{\text {biodiesel }}}{w_{2}^{\text {glicerol }}}
$$

O comportamento do coeficiente de distribuição $\left(K_{2}\right)$ entre as fases ricas em biodiesel e glicerol pode ser observado na Figura 2, onde foi plotado no eixo x a fração mássica do álcool na fase glicerol. 


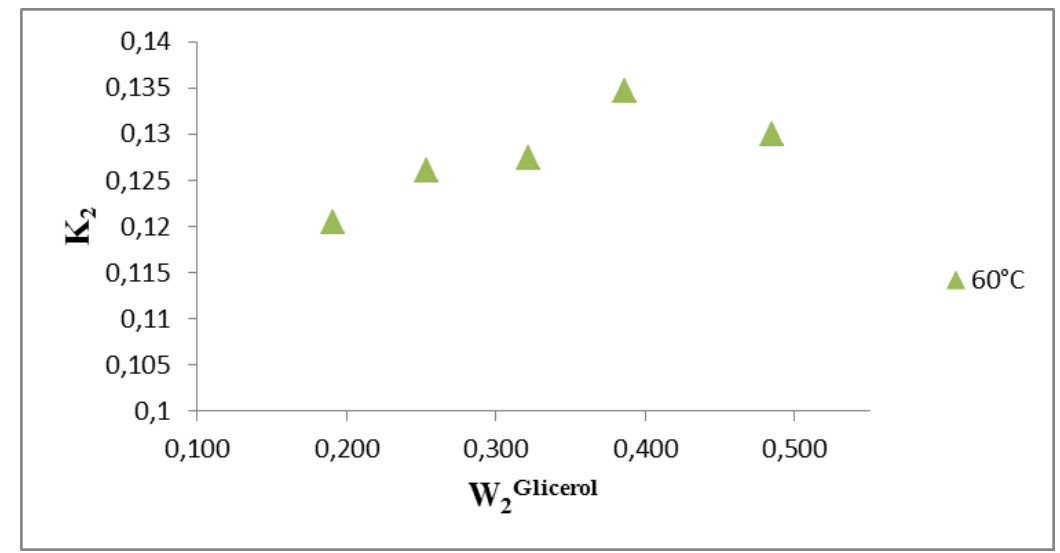

Figura 2 Coeficiente de distribuição para o metanol na fase glicerol a 333,15 K.

Podem-se observar a partir da análise dos coeficientes de distribuição do metanol, que os valores de $\mathrm{K}_{2}$ foram menores do que 1 . Pela definição do coeficiente de distribuição mostrada na equação (3), valores de $\mathrm{K}_{2}<1$ indicam que álcool está distribuído preferencialmente na fase glicerol.

Observa-se na Figura 2, que a maior parte do metanol no sistema biodiesel (1) + metanol (2) + glicerol (3) encontrou-se na fase rica em glicerol, apresentando valores baixos de coeficiente de distribuição. Isto ocorreu devido ao fato de que o metanol e a glicerol apresentam alta polaridade e, consequentemente o metanol distribuiu-se predominantemente na fase glicerol.

A partir das predições realizadas com a metodologia proposta por Stragevitch e d'Ávila, os valores dos parâmetros de interação foram calculados pelo método UNIQUAC, os mesmo são apresentados na Tabela 1.

Tabela 1 Parâmetros globais estimados pelo método UNIQUAC.

\begin{tabular}{cccccc}
\hline $\mathbf{i}$ & $\mathbf{j}$ & $\mathbf{A}(\mathbf{0})_{\mathbf{i j}}$ & $\mathbf{A}(\mathbf{0})_{\mathbf{j i}}$ & $\mathbf{A}(\mathbf{1})_{\mathbf{i j}}$ & $\mathbf{A}(\mathbf{1})_{\mathbf{j i}}$ \\
\hline $\mathbf{1}$ & $\mathbf{2}$ & 65,624 & 451,99 & 0 & 0 \\
$\mathbf{1}$ & $\mathbf{3}$ & 104,16 & 241,62 & 0 & 0 \\
$\mathbf{2}$ & $\mathbf{3}$ & $-288,49$ & 1284,2 & 0 & 0 \\
\hline
\end{tabular}

A Figura 3, construída a partir dos dados das frações mássicas experimentais e com os dados correlacionados através do modelo UNIQUAC, apresenta boa correlação dos dados experimentais e calculados. A exemplo dos trabalhos de MESQUITA et al. (2012), MAZUTTI et al. (2013), PINHEIRO et al. (2014), o modelo UNIQUAC obteve uma boa correlação com os dados experimentais. É importante salientar que todos os trabalhos citados anteriormente foram realizados com biodiesel provenientes de diferentes oleaginosas, assim, o modelo pode ser aplicado para diversos sistemas. 


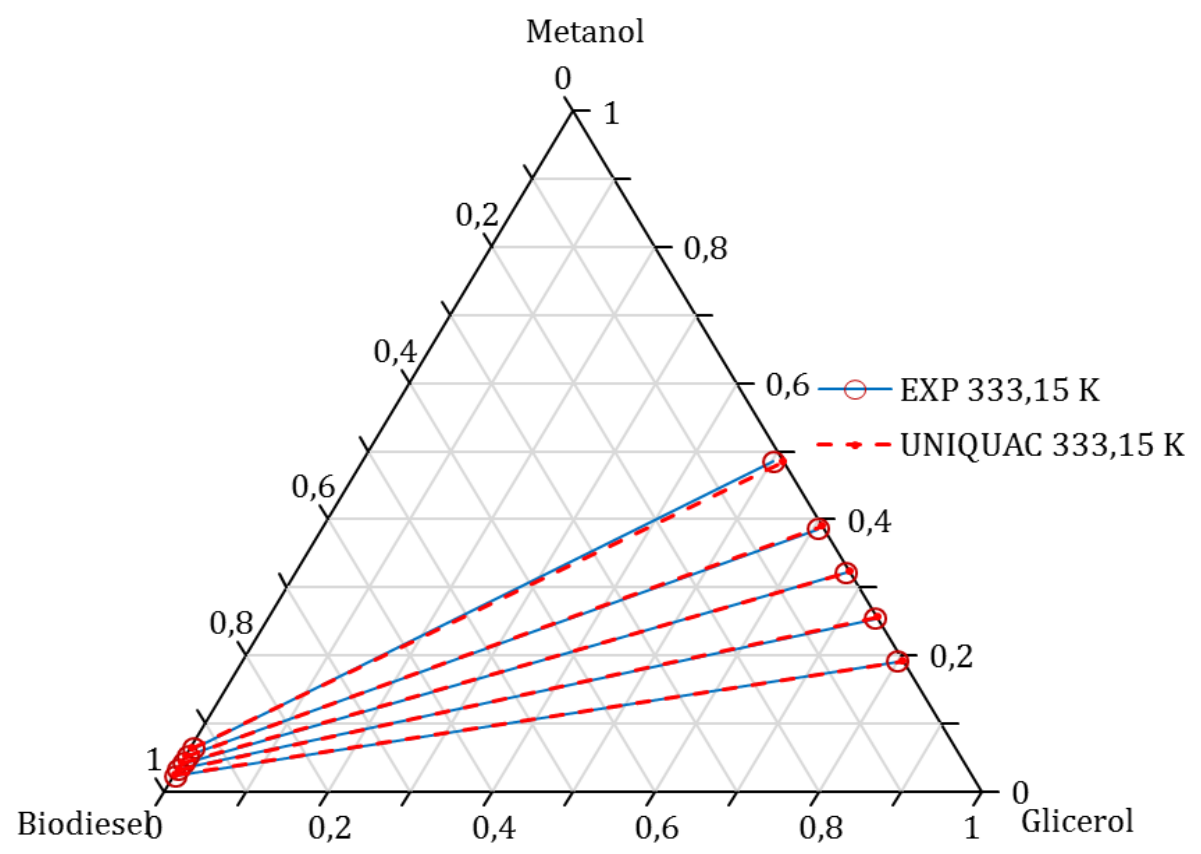

Figura 3 Diagrama para sistema o biodiesel (1) + metanol (2) + glicerol (3) a 333,15 K. Comparação com a correlação utilizando o modelo UNIQUAC.

\section{CONCLUSÕES}

Observou-se que a adição da pequena quantidade de água não apresentou grande influência na separação das fases. Os dados de ELL experimentais foram correlacionados utilizando o modelo UNIQUAC. Para todo o sistema, o desvio global entre os dados calculados e experimentais foi inferior a $0,27 \%$ no modelo UNIQUAC, mostrando a boa qualidade descritiva e aplicabilidade do modelo UNIQUAC na análise do ELL para o sistema avaliado.

\section{REFERÊNCIAS}

ARDILA, Y. C.; MACHADO, A. B.; PINTO, G. M. F; MACIEL, M. R; MACIEL, M. R. W. Liquid-Liquid Equilibrium in Ternary Systems Present in Biodiesel Purification from Soybean Oil and Castor Oil at (298.2 and 333.2) K. J. Chem. Eng. Res. 58, 605-610, (2012).

BALAT, M. BALAT, H., "A critical review of bio-diesel as a vehicular fuel", Energy Conversion and Management 49, 2727-2741, 2008.

FRANÇA, B. B.; VILLARDI, H. G. D; PESSOA, F. L. P., ULLER, A. M. C., Liquid-Liquid Equilibrium for Compounds of Soybean Ethyl Biodiesel Production by Transesterification, J. J. Chem. Eng. Data 2013, 58, 1927-1933. 
LÔBO, I. P. E; COSTA FERREIRA, S. L.; CRUZ, R. S. Biodiesel: Parâmetros de qualidade e métodos analíticos, Química Nova, v.32, n. 6, p. 1596-1608, 2009.

MADURO, R. M. Equilíbrio Líquido-Líquido em Sistemas Nicotina + Água + Extratante. Dissertação de Mestrado, FEQ/UNICAMP, Campinas (2005).

MAIA, A. C. S. ; SILVA, I. S. ; Stragevitch L. . Dados de Equilíbrio Líquido-Líquido para Sistemas de Ésteres Metílicos de Ácido Graxo/Metanol/Glicerol e Ésteres Etílicos de Ácido Graxo/Etanol/Glicerol. In: VI Congresso Brasileiro de Termodinâmica Aplicada, 2011, Salvador. Anais CBTermo 2011.

MAZUTTI, M. A.; VOLL, F. A. P.; CARDOZO-FILHO, L.; CORAZZA, M. L.; LANZA, M.; PRIAMO, W. L.; OLIVEIRA, J. V. Thermophysical properties of biodiesel and related systems: (Liquid + liquid) equilibrium data for soybean biodiesel. J. Chem. Thermodynamics, 58, 83-94, (2013).

MESQUITA, F. M. R. BESSA, A. M. M.; LIMA, D. D. de; SANT'ANA, H. B. de; SantiagoAguiar, R. S. de. Liquid-liquid equilibria of systems containing cottonseed biodiesel + glycerol + ethanol at 293.15, 313.15 and 333.15K. Fluid Phase Equilibria, v. 318 p. 51-55, 2012.

NEGI, D. S.; SOBOTKA, F.; KIMMEL, T.; WONZNY, G.; SCHOMCKER, R. Liquid- Liquid Phase Equilibrium in Glycerol-Methanol-Methyl Oleate and Glycerol-Monoolein-Methyl Oleate Ternary Systems. Ind. Eng. Chem. Res, v. 45, 3693-3696, (2006).

OTHMER, D. T.; TOBIAS, P. E.; Tie Line Correlation, Ind. Eng. Chem., v. 34, 693- 696, (1942).

PINHEIRO, R. S.; BESSA, A. M. M; QUEIROZ, B. A.; DUARTE, A. M. S. F.; SANT'ANA, H. B.; SANTIAGO-AGUIAR, R. S. Optimization of the methylic biodiesel purification process by intermediate of liquid-liquid equilibrium data for ternary systems containing methanol + water + (soybean, corn or brown shell of coconut) biodiesel. Fluid Phase Equilibria. 361 (2014) 30- 36

STRAGEVITCH, L.; D’ÁVILA, S.g.. “Application of a generalized maximum likelihood method in the reduction of multicomponent liquid-liquid equilibrium data." Braz. J. Chem. Eng.. São Paulo, p. 41-52. mar. 1997.

WANG, Y. D.; AL-SHEMMERI, T.; EAMES, P.; MCMULLAN, J.; HEWITT, N.; HUANG Y. "An experimental investigation of the performance and gaseous exhaust emissions of a diesel engine using blends of a vegetable oil". Appl Thermal Eng; 26: 1684-91; 2006. 